\title{
Thyroiditis: A Rare Manifestation of Enasidenib-Induced Differentiation Syndrome
}

\author{
Pavan Annamaraju ${ }^{a} \quad$ Swathi Gopishetty ${ }^{b}$ Naga Goparaju ${ }^{a}$ \\ Matthew Beasey ${ }^{a}$ Vamsi Kota ${ }^{c} \quad$ Achuta K. Guddatic \\ aJohnston Memorial Hospital, Ballad Health System, Abingdon, VA, USA; ${ }^{b}$ Medical Center \\ of Central Georgia, Mercer University, Macon, GA, USA; 'Division of Hematology/Oncology, \\ Georgia Cancer Center, Augusta University, Augusta, GA, USA
}

\section{Keywords}

Acute myeloid leukemia · Chemotherapy · Drug therapy · Enasidenib

\begin{abstract}
Enasidenib is an FDA-approved isocitrate dehydrogenase 2 (IDH2) inhibitor, which is used in the treatment of acute myeloid leukemia (AML). We present a case of AML with an IDH2 mutation treated with a regimen of enasidenib and 5-azacitidine, where thyroiditis was noted to be a part of differentiation syndrome. The patient is a 77-year-old woman with IDH2-mutated AML who had initially been started on $100 \mathrm{mg}$ of enasidenib and then presented with dyspnea and was diagnosed with pleural effusion - a common presentation with enasidenib - but was also noted to have thyroiditis. She was started on steroids, but due to continued hyperbilirubinemia and thyroiditis, her dose of enasidenib was reduced to half, which resulted in clinical improvement. This case demonstrates thyroiditis as one of the rare manifestations in the treatment of AML with enasidenib-induced differentiation syndrome.
\end{abstract}

(C) 2020 The Author(s)

Published by S. Karger AG, Basel

\section{Introduction}

Acute myeloid leukemia (AML) in younger adults has a guarded prognosis, whereas it is significantly worse for older adults and those with secondary AML, which has evolved from antecedent myeloid neoplasms [1]. In patients with relapsed or refractory disease, the 5-year survival rate is only 5-10\% [2]. Enasidenib mesylate is a selective mutant inhibitor of isocitrate dehydrogenase 2 (IDH2) protein, which promotes the differentiation of leukemic 
myoblasts. It was recently approved by the US Food and Drug Administration (FDA) in relapsed/refractory mutant IDH2 AML in 2017 for adults. It is a first-in-class, oral, selective inhibitor of the mutant IDH2 enzyme. Approximately 8-19\% of patients with AML have IDH2 mutations [3]. It has been shown that reductions in IDH2 variant allele frequency and molecular clearance with enasidenib were associated with complete remission, and it also induces responses in approximately $40 \%$ of patients with mutant IDH 2 relapsed/refractory AML regardless of prior treatment [3].

As this is a recently FDA-approved treatment regimen, not many clinical effects of differentiation syndrome have been documented, and, as such, medical literature documenting these effects is crucial to clinicians who treat AML.

\section{Case Presentation}

A 77-year-old Caucasian woman initially presented with pancytopenia, referred from the primary care physician to the hematology/oncology clinic for further evaluation in April 2019. Her past medical history was notable for paroxysmal atrial fibrillation, hyperlipidemia, and recurrent allergic sinusitis. She was on metoprolol, apixaban, and ezetimibe. On presentation, she was afebrile with a blood pressure of 126/80 mm Hg, a heart rate of 72 beats $/ \mathrm{min}$ (regular), and a respiratory rate of 16 breaths/min. A comprehensive physical examination was unremarkable.

The laboratory data revealed pancytopenia with a platelet count of $116 \times 1,000 / \mathrm{mm}^{3}$, a white blood cell count of $1.7 \times 1,000 / \mathrm{mm}^{3}$ with $25 \%$ neutrophils, and a hemoglobin level of $9.5 \mathrm{~g} / \mathrm{dL}$ with a mean corpuscular volume of $80.9 \mathrm{fL} /$ cell and a red blood cell distribution width of 22.9. Flow cytometry was unremarkable. All other laboratory investigations, including a liver function test, creatinine, thyroid function tests, and a hepatitis panel, were also within normal limits. Her iron studies and hemoglobin electrophoresis results were also normal.

She was initially suspected of having $\alpha$-thalassemia, but a bone marrow biopsy in May 2019 demonstrated findings consistent with AML positive for IDH2 mutation with 16\% marrow blasts/promonocytes without circulating blasts. Due to the diagnosis of AML with IDH2 mutation, she was advised against bone marrow transplantation given her age. She was started on a regimen of 5-azacitidine and enasidenib. Prophylactic antiviral, antifungal, and antibiotic medications were also initiated.

After 2 weeks, she was scheduled for the second cycle of chemotherapy. However, it was held off as she reported having subjective fevers associated with headache, frontal sinus pressure, and loss of appetite. Computed tomography of the sinuses showed evidence of inflammatory disease. She was treated with amoxicillin/clavulanic acid (Augmentin) for a presumed diagnosis of bacterial sinusitis. A few days later, her second dose of chemotherapy was administered. During a follow-up visit, her laboratory data revealed hyperbilirubinemia of $3.8 \mathrm{mg} / \mathrm{dL}$, which raised a concern for differentiation syndrome. Therefore, she was started on dexamethasone $8 \mathrm{mg}$ twice a day.

On her next follow-up visit, she complained of dyspnea. Her physical examination was notable for diminished breath sounds at the lung bases and bilateral pitting edema. The chest radiograph confirmed bilateral pleural effusions. A 2D echocardiogram showed a preserved left ventricular ejection fraction. She was treated with oral furosemide along with steroids and pantoprazole. Apixaban was held due to pancytopenia. During this time, she was hospitalized for dehydration in the setting of furosemide use and reduced oral intake. An EKG showed rapid atrial fibrillation. Her laboratory data showed a worsening hyperbilirubinemia up to $5.5 \mathrm{mg} / \mathrm{dL}$, and new-onset hyperthyroidism with TSH less than 
$0.02 \mathrm{ng} / \mathrm{dL}$ and elevated free $\mathrm{T}_{4}$ of $2.1 \mathrm{ng} / \mathrm{dL}$. An I-123 thyroid scan showed slightly increased uptake in the upper portion of the right thyroid lobe with no suppression in the remainder of the gland, consistent with thyroiditis. Rate control of atrial fibrillation was achieved with uptitration of $\beta$-blocker; methimazole was avoided due to concerns for bone marrow suppression.

Due to persistent hyperbilirubinemia despite being on steroids, the dosage of enasidenib was reduced to half. After the dose reduction, repeat total bilirubin started trending down to $4.4 \mathrm{mg} / \mathrm{dL}$. A few weeks later, during a follow-up visit, she was feeling better and was switched to a steroid taper. Her free $\mathrm{T}_{4}$ levels also trended down to $1.5 \mathrm{ng} / \mathrm{dL}$. Subsequently, she started her third cycle of chemotherapy with a reduced dose of enasidenib (50 mg). During a followup visit, her dyspnea and fatigue were noted to have improved and her thyroid panel and bilirubin levels had normalized.

She was able to complete cycles $4 / 5$ and $5 / 5$ of azacytidine with enasidenib uneventfully. Her antifungal and antibiotics were discontinued due to normalization of her absolute neutrophil count.

\section{Discussion}

Differentiation syndrome is an autoimmune response and is associated with fatal complications as it leads to a high mortality rate. AML patients receiving targeted therapy have been shown to have neutrophil recovery in the setting of clinical differentiation syndrome [4]. The differentiation of leukemic blasts and promyelocytes can lead to the release of a systemic inflammatory response leading to cellular migration, endothelial activation, and the release of interleukins (IL-1, IL-6, IL-8, TNF- $\alpha$, and CCL2) and vascular factors which are responsible for tissue damage [5]. Septal edema, intra-alveolar hemorrhage, and capillaritis are seen in the lungs during differentiation syndrome. The symptoms of differentiation syndrome include unexplained fever, weight gain, dyspnea, hypotension, acute respiratory distress with interstitial pulmonary infiltrates, vascular capillary leak syndrome leading to acute renal failure, and hyperbilirubinemia [6]. An elevated white blood cell count is frequently present in differentiation syndrome, but the syndrome can also present with a low white blood cell count, anemia, thrombocytopenia, and coagulopathy. Chest imaging findings are mostly based on the severity of the differentiation syndrome and can present with an increased cardiothoracic ratio, pleural effusion, ground-glass opacity, and pulmonary hemorrhage [7].

Mutant IDH2 proteins neomorphically catalyze the oncometabolite (R)-2-hydroxyglutarate (R-2HG), and high R-2HG concentrations promote hypermethylation, altered gene expression, and blocked differentiation of hematopoietic cells $[8,9]$. Cancer-associated IDH mutations block the normal cellular differentiation via the production of R-2HG. Enasidenib suppresses R-2HG and induces cellular differentiation of mutated IDH2 leukemic cells. Treated cells were noted to have fully functioning neutrophils retaining IDH2 mutation [10]. Pleural effusion is common with enasidenib. This could occur months after initiation of enasidenib treatment and could mimic symptoms of disease progression, and prompt intervention with steroids can be an effective management approach [10]. If not responsive to steroids, an alternative diagnosis should be suspected based on the clinical presentation.

Among patients with relapsed or refractory AML, the overall response rate was $40.3 \%$, with a median response duration of 5.8 months. Median overall survival among relapsed/ refractory patients was 9.3 months, and $19.3 \%$ of patients attained complete remission with an overall survival of 19.7 months [11]. Grade 3-4 enasidenib-related adverse events included 
indirect hyperbilirubinemia (12\%) and IDH inhibitor-associated differentiation syndrome $(7 \%)$ during clinical testing of enasidenib. It appears that instead of cytotoxicity, inducing differentiation is the key mechanism of enasidenib [12].

There have been no reports of thyroid dysfunction in previous clinical studies involving enasidenib. In our patient, differentiation syndrome was suspected, as she presented with dyspnea with pleural effusion and hyperbilirubinemia, and she was started on steroids. She was also noted to have thyroiditis; her symptoms did not respond to the steroid therapy, but later responded to a decreased dose of enasidenib. This report underlines the importance of considering thyroiditis as a possible manifestation of differentiation syndrome in AML patients being treated with enasidenib.

\section{Statement of Ethics}

The patient had consented for her clinical history and case details to be published.

\section{Disclosure Statement}

No conflicts of interest exist for any of the authors.

\section{Funding Sources}

The authors declare that there was no funding for this study.

\section{Author Contributions}

P. Annamaraju, S. Gopishetty, N. Goparaju, V. Kota, and A.K. Guddati: literature review, and writing and revision of the manuscript; M. Beasey: literature review and revision of the manuscript.

\section{References}

1 Walter RB, Estey EH. Management of older or unfit patients with acute myeloid leukemia. Leukemia. 2015; 29(4):770-5.

2 Oran B, Weisdorf DJ. Survival for older patients with acute myeloid leukemia: a population-based study. Haematologica. 2012;97(12):1916-24.

3 Stein EM, DiNardo CD, Fathi AT, Pollyea DA, Stone RM, Altman JK, et al. Molecular remission and response patterns in patients with mutant-IDH2 acute myeloid leukemia treated with enasidenib. Blood. 2019; 133(7):676-87.

4 Birendra KC, DiNardo CD. Evidence for clinical differentiation and differentiation syndrome in patients with acute myeloid leukemia and IDH1 mutations treated with the targeted mutant IDH1 inhibitor, AG-120. Clin Lymphoma Myeloma Leuk. 2016;16(8):460-5.

5 Luesink M, Jansen JH. Advances in understanding the pulmonary infiltration in acute promyelocytic leukaemia. Br J Haematol. 2010;151(3):209-20.

6 Montesinos P, Sanz MA. The differentiation syndrome in patients with acute promyelocytic leukemia: experience of the PETHEMA group and review of the literature. Mediterr J Hematol Infect Dis. 2011;3(1):e2011059.

7 Jung JI, Choi JE, Hahn ST, Min CK, Kim CC, Park SH. Radiologic features of all-trans-retinoic acid syndrome. AJR Am J Roentgenol. 2002;178(2):475-80.

8 Losman JA, Kaelin WG Jr. What a difference a hydroxyl makes: mutant IDH, (R)-2-hydroxyglutarate, and cancer. Genes Dev. 2013;27(8):836-52. 
9 Figueroa ME, Abdel-Wahab O, Lu C, Ward PS, Patel J, Shih A, et al. Leukemic IDH1 and IDH2 mutations result in a hypermethylation phenotype, disrupt TET2 function, and impair hematopoietic differentiation. Cancer Cell. 2010;18(6):553-67.

10 Fathi AT, DiNardo CD, Kline I, Kenvin L, Gupta I, Attar EC, et al. Differentiation syndrome associated with enasidenib, a selective inhibitor of mutant isocitrate dehydrogenase 2: analysis of a phase 1/2 study. JAMA Oncol. 2018;4(8):1106-10.

11 Stein EM, DiNardo CD, Pollyea DA, Fathi AT, Roboz GJ, Altman JK, et al. Enasidenib in mutant IDH2 relapsed or refractory acute myeloid leukemia. Blood. 2017;130(6):722-31.

12 Stein EM. Enasidenib, a targeted inhibitor of mutant IDH2 proteins for treatment of relapsed or refractory acute myeloid leukemia. Future Oncol. 2018;14(1):23-40. 